\title{
Photomixers fabricated on nitrogen-ion-implanted GaAs
}

\author{
M. Mikulics ${ }^{\mathrm{a})}$ \\ Max-Planck-Institute for Radioastronomy, Bonn, D-53121 Bonn, Germany, and Institute of Thin Films and \\ Interfaces, Research Center Jülich, D-52425 Jülich, Germany \\ M. Marso \\ Institute of Thin Films and Interfaces, Research Center Jülich, D-52425 Jülich, Germany \\ I. Cámara Mayorga and R. Güsten \\ Max-Planck-Institute for Radioastronomy, Bonn, D-53121 Bonn, Germany \\ S. Stanček and P. Kováč \\ Department of Nuclear Physics and Technology, Slovak University of Technology, Bratislava, Slovakia \\ S. Wu, ${ }^{\text {b) }}$ Xia Li, ${ }^{\text {c) }}$ M. Khafizov, ${ }^{\text {b) }}$ and R. Sobolewski ${ }^{\mathrm{c})}$ \\ Department of Electrical and Computer Engineering and the Laboratory for Laser Energetics, University of \\ Rochester, Rochester New York 14627-0231
}

E. A. Michael and R. Schieder

1. Physikalisches Institut der Universität zu Köln, D-50937 Köln, Germany

M. Wolter, D. Buca, A. Förster, P. Kordoš, and H. Lüth

Institute of Thin Films and Interfaces, Research Center Jülich, D-52425 Jülich, Germany

(Received 31 January 2005; accepted 15 June 2005; published online 20 July 2005)

\begin{abstract}
We report on fabrication and measurement of photomixers based on nitrogen-ion-implanted GaAs. We used energies of $500 \mathrm{keV}, 700 \mathrm{keV}$, and $880 \mathrm{keV}$ to implant $\mathrm{N}^{+}$ions into GaAs substrates with an ion concentration of $\sim 3 \times 10^{12} \mathrm{~cm}^{-2}$. The resulting material exhibited $110 \mathrm{fs}$ carrier lifetime due to implantation-induced defects. Our photomixers were fabricated as metal-semiconductor-metal devices, placed at the feed point of a broadband antenna. Optoelectronic measurements were performed in the wavelength range between $350 \mathrm{~nm}$ and $950 \mathrm{~nm}$. In comparison to their counterparts (photomixers fabricated on low-temperature-grown GaAs) the $\mathrm{N}^{+}$-implanted GaAs photomixers exhibit improvements on both the output power and responsivity. A maximal responsivity of above $100 \mathrm{~mA} / \mathrm{W}$ was achieved and we did not observe any dependence of the mixer cut-off frequency on the bias voltage. These characteristics make $\mathrm{N}^{+}$-implanted GaAs the material of choice for efficient optoelectronic photomixers. () 2005 American Institute of Physics. [DOI: $10.1063 / 1.2006983$ ]
\end{abstract}

Ion implantation is well known to reduce carrier lifetime in GaAs-based photodetectors. Values in the picosecond and even subpicosecond regime have been reported in the past. The properties of GaAs implanted with protons, ${ }^{1} \mathrm{Ar}^{+}, 2,3$ $\mathrm{As}^{+},{ }^{4-6}$ and other ions have been extensively investigated. The implantation of nitrogen ions in GaAs, however, is relatively new. The initial objective of implanting $\mathrm{N}$ ions in GaAs was to get the diluted ternary semiconductor GaAsN. The optical properties of this new material, such as photoluminescence ${ }^{7}$ and $\mathrm{N}^{+}$-induced band-gap reduction, ${ }^{8}$ have been studied in previous works. It is also noted that high-energy $\mathrm{N}^{+}$-implanted GaAs becomes a highly resistive material after high-temperature annealing., ${ }^{9,10}$

In a previous work, we have presented ultrafast photodetectors based on $\mathrm{N}^{+}$-implanted GaAs with increased responsivity and very low dark currents. ${ }^{11}$ The purpose of this letter is to describe the fabrication process, as well as the properties of photomixers fabricated using high energy

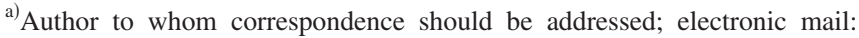
m.mikulics@fz-juelich.de

${ }^{b)}$ Also at: Department of Physics and Astronomy, University of Rochester, Rochester, NY 14627.

c) Also at: Materials Science Program, University of Rochester, Rochester, NY 14627.
}

$\mathrm{N}^{+}$-implanted GaAs substrates. Furthermore, we present a performance improvement of these photomixers, as to the devices fabricated on low-temperature-grown GaAs (LT-GaAs). ${ }^{12-14}$

The fabrication process started with implantation of $\mathrm{N}$ ions with energies of $500 \mathrm{keV}, 700 \mathrm{keV}$, and $880 \mathrm{keV}$, respectively, into $2 \mu \mathrm{m}$ thick, $10^{17} \mathrm{~cm}^{-3} n$-doped GaAs layers grown by molecular-beam epitaxy on semi-insulating GaAs (001) substrates. The implantation was performed by the linear, $900 \mathrm{kV}$ accelerator at the Slovak University of Technology, Bratislava, Slovakia. For all samples, the ion dose was about $3 \times 10^{12} \mathrm{~cm}^{-2}$. The depth of the implantation maximum was about $800 \mathrm{~nm}, 1000 \mathrm{~nm}$, and $1200 \mathrm{~nm}$ for the 500, 700 , and $880 \mathrm{keV}$ implantation energies, respectively. ${ }^{11,15}$ Next, metal-semiconductor-metal (MSM) structures with an active area of $100 \mu \mathrm{m}^{2}$ and finger width and spacing of $1 \mu \mathrm{m}$ and $1.5 \mu \mathrm{m}$, respectively, were patterned on the $\mathrm{N}^{+}$-implanted GaAs materials using conventional photolithography and lift-off technique. ${ }^{10,11}$ The MSM structures consisted of $\mathrm{Ti} / \mathrm{Au}$ contacts with 10/160 nm thicknesses. For comparison, an MSM structure with the same geometry as above was fabricated on LT-GaAs. After fabrication of the MSM structures, their whole surface was coated with $200 \mathrm{~nm}$ of $\mathrm{SiO}_{2}$ and the windows were opened in the $\mathrm{SiO}_{2}$ layer to contact the MSM electrodes. Ti/Au bow-tie anten- 


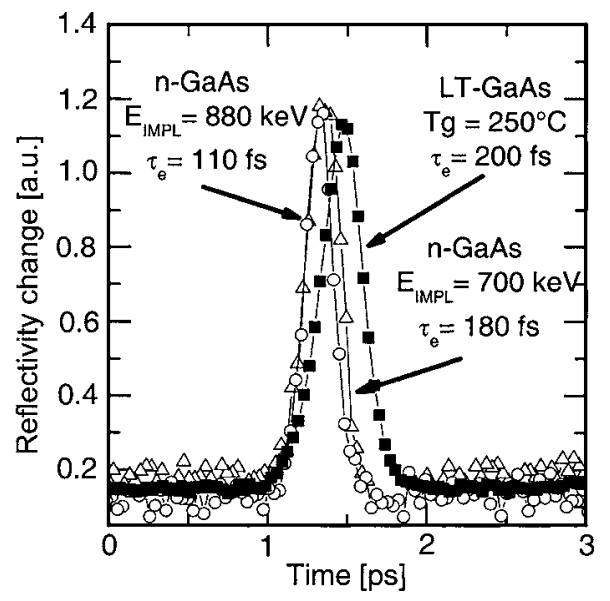

FIG. 1. Carrier lifetimes in LT-GaAs (growth temperature $T_{g}=250{ }^{\circ} \mathrm{C}$ ) and in $\mathrm{N}^{+}$-implanted GaAs implanted with energies $700 \mathrm{keV}$ and $880 \mathrm{keV}$, obtained from the normalized optical reflectivity change measured using femtosecond pump/probe spectroscopy.

nas with thicknesses of 50/600 nm were fabricated on the top of the $\mathrm{SiO}_{2}$ insulator layer.

The lifetime of the photogenerated carriers in $\mathrm{N}^{+}$-implanted GaAs and LT-GaAs was studied using femtosecond time-resolved reflectivity measurements by an alloptical pump/probe system featuring $\sim 70$ fs temporal resolution. Carrier lifetimes of less than 200 fs were obtained for the samples implanted with energies in the range from $500 \mathrm{keV}$ up to $880 \mathrm{keV} .{ }^{10}$ Figure 1 shows our experimental pump/probe results for both $\mathrm{N}^{+}$-implanted GaAs and LTGaAs. We note that the material implanted with $880 \mathrm{keV}$ energy exhibits a carrier lifetime of only $110 \mathrm{fs}$, which is almost $50 \%$ shorter than the value measured for LT-GaAs. Significantly shorter carrier lifetime translates in wider bandwidth and better $\mathrm{THz}$ efficiency of the photomixers based on $\mathrm{N}^{+}$-implanted GaAs, as compared to those based on LT-GaAs. ${ }^{12}$ The other very important feature of photomixers is their as high as possible photocurrent, or high responsivity, in order to assure the highest possible output power. The photodetectors based on $\mathrm{N}^{+}$-implanted GaAs exhibit more than twice the responsivity when compared to LT-GaAs devices, as was demonstrated in our previous works. ${ }^{10,11}$

Figure 2 depicts the results of our spectral responsivity measurements performed with MSM photodetectors based

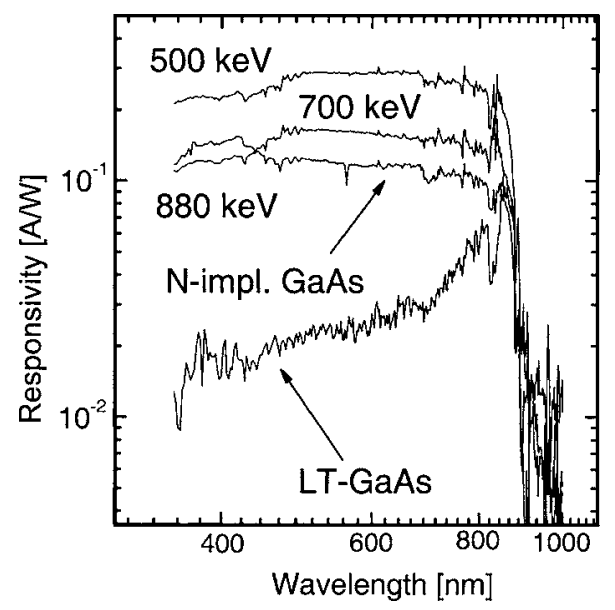

FIG. 2. Spectral responsivity characteristics for photomixers fabricated on LT-GaAs and $\mathrm{N}^{+}$-implanted GaAs materials, measured at $10 \mathrm{~V}$ bias voltage.

Downloaded 21 Dec 2006 to 134.94.122.39. Redistribution subjec

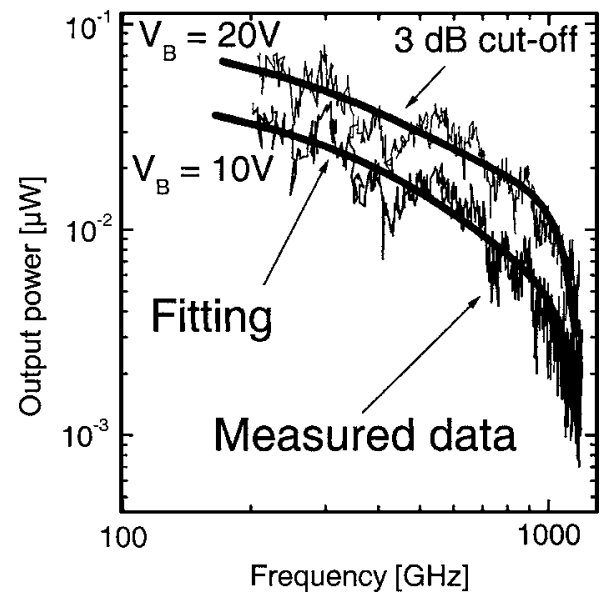

FIG. 3. Output power (thin lines) for a photomixer fabricated on $\mathrm{N}^{+}$-implanted $\mathrm{GaAs}$ as a function of the intermediate frequency, measured for $780 \mathrm{~nm}$ wavelength, $70-80 \mathrm{~mW}$ input power optical radiation for two bias levels $(10 \mathrm{~V}$ and $20 \mathrm{~V})$. Implantation energy is $880 \mathrm{keV}$. Bold solid lines correspond to a polynomial fit as a guide for the eyes.

on $\mathrm{N}^{+}$-implanted GaAs and compared with those based on LT-GaAs. The spectral measurements were performed with a setup where the light from a xenon arc lamp is directed through a grating monochromator. The monochromatic light is focused by a lens to the device under test. The measurement accuracy is improved by a lock-in amplifier including a chopper in the light beam. The responsivity increases with a decrease of the implantation energy. This dependence is due to the shorter implantation depth for lower energies. In this case, a higher amount of photons penetrates the nonmodified bulk material with larger carrier lifetime. The photoconductor gain and therefore the responsivity are proportional to the lifetime of the photogenerated carriers, resulting in the higher responsivity for the lower energy devices. Figure 2 also shows that the responsivity of photodetectors based on the implanted material increases at shorter wavelengths, in contrast to the results obtained for LT-GaAs. We explain this behavior by the smaller penetration depth of the shortwavelength light. It decreases from $1 \mu \mathrm{m}$ at $850 \mathrm{~nm}$ wavelength to $100 \mathrm{~nm}$ at $500 \mathrm{~nm}$ wavelength. ${ }^{16}$ A larger amount of the photocurrent is then generated near the surface where the defect density is smaller, giving a larger quantum efficiency. This increase of the quantum efficiency with decreasing wavelength compensates for the usual linear dependence of the responsivity on wavelength.

Photomixing measurements were performed in a heterodyne photomixer setup with 780-nm-wavelength continuouswave radiation from two solid-state lasers. ${ }^{17}$ Experimental data shown in Fig. 3 demonstrate that devices on $\mathrm{N}^{+}$-implanted GaAs can indeed, very efficiently, generate $\mathrm{THz}$ radiation. The output power depends on the bias voltage, as it was expected by the voltage-dependent responsivity of photoconductors ${ }^{11}$ and reached $13 \mathrm{nW}$ at $1 \mathrm{THz}$ at the bias voltage of $20 \mathrm{~V}$. The fitting curve corresponds to a polynomial fit as a guide for the eyes. The gradual rolloff between 100 and $1000 \mathrm{GHz}$ and the faster roll-off above $1 \mathrm{THz}$ in the input power is due to the two low-pass contributions by carrier lifetime and resistance/capacitance time constant. ${ }^{12}$ A further increase of the output power could be obtained by the optimization of the photodetector design to a smaller active area with sub- $\mu \mathrm{m}$ MSM finger widths, and by using a dipole-type instead of the bow-tie-type THz antenna.
to AIP license or copyright, see http://apl.aip.org/apl/copyright.jsp 


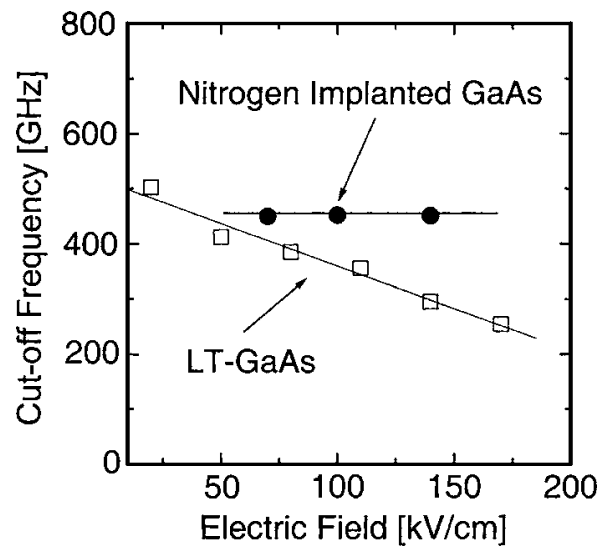

FIG. 4. The cut-off frequency dependencies as a function of the applied electric field for $\mathrm{N}^{+}$-implanted GaAs (implantation energy: $880 \mathrm{keV}$; closed circles) and LT-GaAs (open squares) photomixers.

Finally, Fig. 4 shows the dependence of the cut-off frequency ( $3 \mathrm{~dB}$ drop from the low-frequency value) of our $\mathrm{N}^{+}$-implanted GaAs photomixers on the average electric field defined as the ration of applied bias to MSM finger spacing, again, in comparison with LT-GaAs devices. It should be noted that the effective electric field in the depth of the implantation maximum decreases to about $15 \%$ of the above mentioned average value. ${ }^{18}$ For the LT-GaAs photomixers, the cut-off frequency decreases rapidly with the increase of the applied electric field, while in the case of the $\mathrm{N}^{+}$-implanted GaAs photomixers the cut-off frequency does not depend on the applied bias and remains constant in the whole range of tested electric fields (between 50 and $200 \mathrm{kV} / \mathrm{cm}$ ). The behavior observed in the LT-GaAs devices is typical for their performance and indicates a reduction of the electron capture cross section with the increased electric field, as was observed earlier by Zamdmer and Hu. ${ }^{19}$ Our $\mathrm{N}^{+}$-implanted GaAs photomixers are clearly free of the problem of the rf power saturation with increasing bias. We attribute this superior behavior of the implanted material to the different, as compared to LT-GaAs, physical origin of the implantation defects that act as carrier traps.

We have fabricated $\mathrm{THz}$ bandwidth photomixers based on $\mathrm{N}^{+}$-implanted GaAs. The different origin of the carrier traps, formed during the implantation process, as compared to As precipitates on LT-GaAs lead to the novel material with ultrashort carrier lifetime and, simultaneously, increased responsivity, leading to the very good performance of photomixers fabricated on the implanted material. As compared to photomixers fabricated on LT-GaAs, our devices show higher cut-off frequencies for electric fields in the range from 50 to $200 \mathrm{kV} / \mathrm{cm}$. Simultaneously, the value of the cut-off frequency is independent of the bias voltage, which eliminates the problem of the rf output power saturation, typical for the LT-GaAs-based photomixers. A further improvement of the device performance is expected by the fine-tuned optimization of the implantation dose and energy of the nitrogen ions and by fabrication of MSM structures with submicrometer dimensions.

This work was supported in part by the Ministry of Education of the Slovak Republic via grants VEGA No. 2/5130/25 and VEGA No. 1/0274/03. Additional support (Rochester) came from the NSF Grant No. INT-0078949, Corning Inc., and NYSTAR through the Center for Electronic Imaging Systems at the University of Rochester. One of the authors (S. W.) acknowledges support from the Frank Horton Graduate Program in Laser Energetics.

${ }^{1}$ M. Lambsdorff, J. Kuhl, J. Rosenzweig, A. Axmann, and J. Schneider, Appl. Phys. Lett. 58, 1881 (1991).

${ }^{2}$ A. A. Kutas, T. V. Kovyazina, A. N. Akimov, G. A. Gusakov, F. F. Komarov, A. P. Novikov, and L. A. Vlasukova, Mater. Sci. Eng., B 34, 32 (1995).

${ }^{3}$ B. Breeger, E. Wendler, C. Schubert, and W. Wesch, Nucl. Instrum. Methods Phys. Res. B 161, 415 (2000).

${ }^{4}$ A. Claverie, F. Namavar, and Z. Liliental-Weber, Appl. Phys. Lett. 62, 1271 (1993).

${ }^{5}$ F. Ganikhanov, G.-R. Lin, W.-C. Chen, C.-S. Chang, and C.-L. Pan, Appl. Phys. Lett. 67, 3465 (1995).

${ }^{6}$ H. Fujioka, J. Krueger, A. Prasad, X. Liu, and E. R. Weber, J. Appl. Phys. 78, 1470 (1995).

${ }^{7}$ X. Weng, S. J. Clarke, W. Ye, S. Kumar, R. S. Goldman, A. Daniel, R. Clarke, J. Holt, J. Sipowska, A. Francis, and V. Rotberg, J. Appl. Phys. 92, 4012 (2002).

${ }^{8}$ W. Shan, K. M. Yu, W. Walukiewicz, J. W. Ager III, E. E. Haller, and M. C. Ridgway, Appl. Phys. Lett. 75, 1410 (1999).

${ }^{9}$ J. F. Chen, J. S. Wang, M. M. Huang, and N. C. Chen, Appl. Phys. Lett. 76, 2283 (2000).

${ }^{10}$ M. Mikulics, PhD thesis, RWTH Aachen and Research Center Juelich, 2005.

${ }^{11}$ M. Mikulics, M. Marso, P. Kordoš, S. Stanček, P. Kováč, X. Zheng, S. Wu, and R. Sobolewski, Appl. Phys. Lett. 83, 1719 (2003).

${ }^{12}$ E. R. Brown, K. A. McIntosh, F. W. Smith, M. J. Manfra, and C. L. Dennis, Appl. Phys. Lett. 62, 1206 (1993).

${ }^{13}$ K. A. McIntosh, E. R. Brown, K. B. Nichols, O. B. McMahon, W. F. diNatale, and T. M. Lyszczarz, Appl. Phys. Lett. 67, 3844 (1995).

${ }^{14}$ E. Peytavit, S. Arscott, D. Lippens, G. Mouret, S. Matton, P. Masselin, R. Bocquet, J. F. Lampin, L. Desplanque, and F. Mollot, Appl. Phys. Lett. 81, 1174 (2002).

${ }^{15}$ J. F. Ziegler, J. P. Biersack, and U. Littmark, The Stopping and Range of Ions in Solids (Pergamon, New York, 1985), Vol. 1; see also http:// www.srim.org/

${ }^{16}$ D. K. Schroeder, Semiconductor Material and Device Characterization (Wiley, New York, 1990).

${ }^{17}$ I. C. Mayorga, M. Mikulics, A. Schmitz, P. Van der Wal, R. Güsten, M. Marso, P. Kordoš, and H. Lüth, Proc. SPIE 5498, 537 (2004).

${ }^{18}$ E. R. Brown, Appl. Phys. Lett. 75, 769 (1999).

${ }^{19}$ N. Zamdmer and Q. Hu, Appl. Phys. Lett. 75, 2313 (1999). 